\title{
La informalidad empresarial y las prácticas administrativas en empresas constructoras de Colombia
}

\section{Informal Businesses and administrative practices in construction companies in Colombia}

Págs. 9-16

Grupo de Investigación: Gestion y Competitividad de las Organizaciones

Línea de investigación Competitividad de empresas constructoras

Rodrigo Andrés Ruiz Medina•

\section{RESUMEN}

El presente artículo es el punto de partida de un proyecto investigativo que tiene como fin identificar algunas prácticas administrativas o de gestión en empresas informales del sector de la construcción. Y en este sentido, define el concepto de práctica administrativa que será usado en él. Posteriormente, realiza una primera aproximación al enfoque administrativo reinante en este tipo de empresas, resaltando el grado de informalidad percibido en las mismas y vinculándolo con algunas estadísticas nacionales, las cuales muestran los índices de informalidad según un estudio realizado en la Encuesta Nacional de Micro-establecimientos.

Palabras clave: informalidad empresarial, construcción, administración, gestión, prácticas administrativas.

\begin{abstract}
This article is the starting point of a research project that aims to identify some administrative or management practices in informal enterprises at the construction sector. And in this sense, it defines the concept of administrative practice to be used in it. Then it makes a first approach to the prevailing administrative approach in these kind of enterprises, highlighting the perceived degree of informality and linking them with some national statistics, which show rates of informality according to a study in the National Survey of Micro- establishments.
\end{abstract}

Keywords: informal businesses, construction, administration, management, management practices

\footnotetext{
- Ingeniero Químico, Docente Investigador, Fundación Universidad de América. Correo electrónico: rodrigo.ruiz@investiga-
} dores.uamerica.edu.co 


\section{INTRODUCCIÓN}

Qué es gestión. Para abordar el concepto de gerencia empírica, es importante primero abordar algunas definiciones para posteriormente contextualizar el estudio de acuerdo con la pregunta problema.

Gerencia, gestión y administración son definiciones globalmente aceptadas y utilizadas en el ámbito académico, sin embargo, hay diferencias sutiles que han generado diversas posturas en el contexto de las ciencias sociales y económicas.

En los años cuarenta la gerencia era concebida como administración de máquinas e infraestructura principalmente, pero a partir de los años cincuenta se empezó a hablar de gestión de recursos humanos y posteriormente de gestión del conocimiento.

Krygier (1992) la define la gerencia como un macro concepto que integra la organización como entidad, sus procesos dinámicos e interactivos, la viabilidad de esos procesos para alcanzar objetivos, y la capacidad que esta tiene para asegurar su supervivencia y desarrollo, empleando en forma eficaz los recursos de los cuales dispone. Crosby (2011) define la gerencia como "el arte de hacer que las cosas ocurran". Según Paulo Roberto Motta (1993) la gerencia es una acción científica, que involucra el análisis de relaciones causa-efecto en aras de la anticipación y la prevención.

Si se revisan estas y otras definiciones académicas, cuando se refieren al concepto o función de gerencia se puede entender como un proceso que implica la coordinación de los recursos disponibles en una organización (humano, físico, tecnológico, financiero, información), para que a través de diversos procesos, se alcancen objetivos planteados. De acuerdo con lo anterior se pueden identificar tres niveles claves de intervención en las que se pueden caracterizar las actividades de la gerencia en un marco de macro-procesos. En primer lugar, la planeación y asignación estratégica de recursos en la organización; en segundo lugar, la ejecución u operación y en tercer lugar, las actividades de soporte o apoyo.

Qué es Administración. La palabra administración proviene del latín ministerium que significa servicio, empleo u oficio, y se asocia al minister o servidor oficial (Corominas y Pascual, 1984).

Desde la década de 1920, con los aportes de Frederick Taylor(1977) y Henry Fayol(1947), implícitamente se hace referencia al proceso de planear, organizar, dirigir y controlar los esfuerzos de los miembros de la organización, y de administrar los demás recursos de ésta, para alcanzar metas establecidas (Stoner, Freeman, Sacristan y Gilbert, 1996).

Aunque la palabra gestión procede del Latín Gestio-Gestionis y abriga conceptualmente la ejecución exitosa con medios pertinentes (Corominas y Pascual, 1984), desde la década del cincuenta, Peter Drucker (2008) la redefine como una función y a la vez una persona sobre la que recae cierta responsabilidad, denotando una posición de autoridad pero a su vez, una disciplina o campo de estudio.

Chung y Megginson (1981) establecen dos niveles de trabajo gerencial, que dan respuesta al enfoque dialógico organizacional contemporáneo. Por un lado se encuentra el aspecto normativo, referente a lo que la organización "espera" que haga el gerente y que ha sido asociado a las funciones clásicas de la administración señaladas en la década del veinte como la planificación, la orga- 
LÍNEA DE INVESTIGACIÓN: COMPETITIVIDAD DE EMPRESAS CONSTRUCTORAS

nización, la coordinación y el control; y por el otro, el enfoque descriptivo que se centra en las otras actividades que un gerente ejecuta. 1. Actividades personales como la distribución de su tiempo, el desarrollo de su propia carrera, y el manejo de sus asuntos. 2. Actividades de interacción de tipo directivo, informacional y decisional. 3. Actividades administrativas relacionadas con documentación, evaluación de políticas y procedimientos y preparación y administración de presupuestos. Y 4. Actividades técnicas que involucran el uso de herramientas y el ejercicio de habilidades para la solución de problemas.

\section{MARCO TEÓRICO}

\section{El enfoque administrativo de la pyme constructora}

A partir de una entrevista semiestructurada realizada a un grupo de veinte gerentes de pymes constructoras, se pudo indagar acerca de los estilos administrativos y de gestión de algunas de estas empresas. De tal manera se identificaron una serie de características propias de este tipo de organizaciones en relación al sector en el que se encuentran inmersas.

De acuerdo con lo indagado, en primer lugar, las pymes constructoras se conciben como empresas que basan su actividad económica en el desarrollo de proyectos, pues sus productos son el resultado natural de la formulación y ejecución de obras constructivas, lo cual hace que todos los procesos administrativos, operativos y de apoyo estén orientados a una gestión por lotes y con estacionalidades determinadas por el número de proyectos a ejecutar en el año fiscal.

Esta dinámica empresarial incentiva un movimiento de contracción y expansión constante de tamaño de empresa o de operación, que incrementa o reduce recursos financieros, humanos y físicos según el período por el que se transita, obligando así a concentrar los esfuerzos en la dimensión técnico-operacional puesto que es la clave del éxito para la supervivencia en el mercado.

Otra particularidad de la empresa constructora que se identifica en este estudio exploratorio, es la generación de excedentes o rentabilidades al final de cada proyecto, lo cual crea en la cultura de la empresa una sensación de abundancia y exceso de liquidez, anquilosa a la organización en una zona de confort e induce a la desaceleración del desempeño global o en caso extremo, a la inactividad o cierre total de la empresa por malos manejos financieros.

Cuando dicha abundancia se desdibuja en el mediano plazo y la empresa logra reaccionar, se ve obligada a reactivar todas las actividades entorno a un nuevo proyecto que garantice otra vez abundancia temporal. De esta manera, se genera un ciclo estacional que se puede repetir 3 o 4 veces en un año de operación.

Esta dinámica de las pymes constructoras produce un descuido generalizado en las actividades de gestión empresarial, haciendo casi inexistentes las funciones de planeación y control corporativo, y reduciendo estas a dimensiones técnico-operativas, lo que propicia informalidad en diferentes departamentos o procesos. 


\section{EL CONCEPTO DE INFORMALIDAD EMPRESARIAL}

Santamaría y Rozo (2009) adelantaron un estudio de informalidad empresarial en Colombia, en el cual infieren que ésta se rige por la ausencia de documentos legales, y asociado a ella, el incumplimiento de las obligaciones legales de la empresa.

La OECD (Development. Statistics Directorate, International Monetary Fund. Bureau of Statistics, International Labour Organisation. Bureau of Statistics, \& Commonwealth of Independent States. Statistical Committee, 2002) propone métodos directos e indirectos para la medición de informalidad empresarial, y aunque el método indirecto fue aceptado y validado por el grado de aproximación en el cálculo de variables como cantidad de demanda eléctrica, número de notas bancarias transadas, etc., se considera que el método indirecto tiene mayor efectividad en las estimaciones.

Pedersen (2003) utiliza el método directo en un estudio que adelanta en Alemania, Gran Bretaña y Escandinavia, revelando que del total del trabajo informal, el $46,7 \%$ es encontrado en el sector de la construcción. Estos hallazgos exponen el carácter global de la problemática de la informalidad empresarial.

Por otro lado, Hamann y Mejía (2011) en un estudio realizado en Colombia con datos recolectados con la Encuesta Nacional de Micro establecimientos en el período comprendido entre el 2000 y el 2007, introdujeron la combinación de tres criterios que una empresa en operación debe cumplir para ser considerada como formal.

(1) El establecimiento debe llevar contabilidad, (2) el establecimiento debe tener un registro mercantil vigente (RM), y (3) el establecimiento debe pagar prestaciones sociales y contribuciones a la nómina (PSS)

Bajo estos criterios los autores definen cuatro grados de formalidad:

"Grado I (informalidad total): no contabilidad, no RM y no PSS.

Grado II (informalidad parcial): sí contabilidad o sí RM o sí PSS.

Grado III (formalidad parcial): (sí contabilidad y sí RM) ó (sí contabilidad y sí PSS) ó (sí RM y sí

PSS), y

Grado IV (formalidad total): sí contabilidad, sí RM y sí PSS". (Hamann y Mejía, 2011, p. 7)

\section{LA INFORMALIDAD EMPRESARIAL EN COLOMBIA}

Con base en esta clasificación, Hamann y Mejía, obtuvieron los resultados presentados en la figura 1: 
LÍNEA DE INVESTIGACIÓN: COMPETITIVIDAD DE EMPRESAS CONSTRUCTORAS

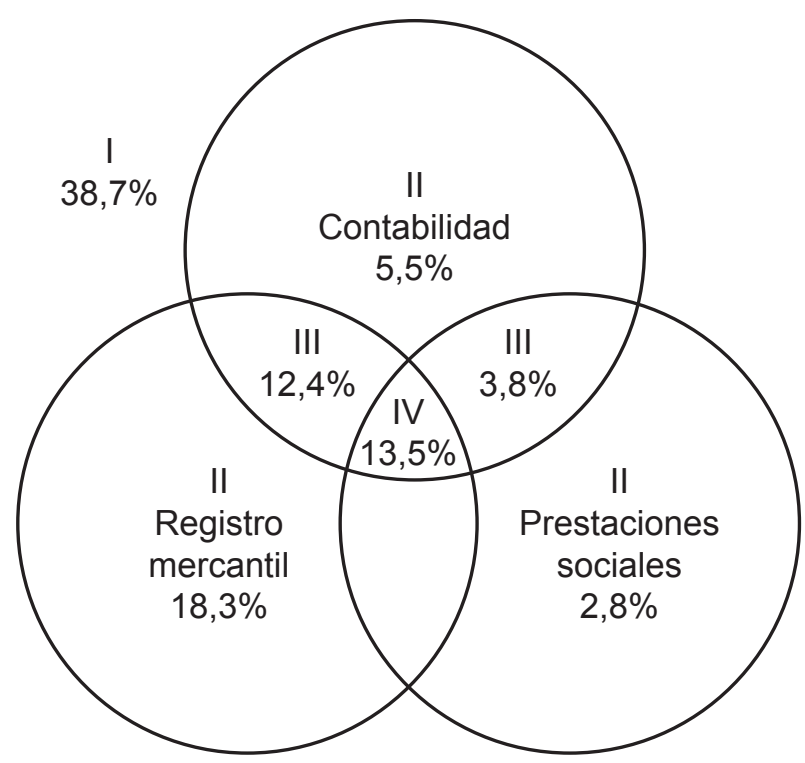

Figura 1. Informalidad Empresarial en micro-establecimientos

Fuente: Hamann y Mejía. (2011)

El 38,7\% de las empresas que participaron en el estudio se encuentran en la informalidad total, el $5,5 \%$ no presentan actividad contable alguna, el $18,3 \%$ no presentan registro mercantil y el $2,8 \%$ no presentan pagos de prestaciones sociales.

Lo interesante del estudio es la combinación de factores que evidencia cómo el $12,4 \%$ de las empresas no presentan registro mercantil ni contabilidad, y solamente el $13,5 \%$ del total de la muestra se encuentra en un estado total de formalidad, lo cual presenta un nivel muy bajo en la formalidad empresarial en Colombia.

Estos resultados obligan a plantear como hipótesis que el $86,5 \%$ de las empresas están utilizando algún grado de informalidad en sus prácticas administrativas o de gestión, lo que amerita adelantar una investigación a nivel sectorial que explore dicho fenómeno.

\section{MÉTODO}

La investigación realizada es de tipo exploratoria dado que Esta hace parte de un proyecto de mayor envergadura que demanda en su primer estado, una aproximación a dos de los conceptos más relevantes, como lo son la informalidad empresarial y las prácticas administrativas, y su relación en el contexto de empresas constructoras.

El corte de esta primera fase es de tipo cualitativo, documental y centrada en un análisis transversal dado que busca comparar la informalidad en empresas del sector de la construcción, e identificar si dentro de la literatura, existen referencias de medición de prácticas administrativas en este tipo de empresas. 


\section{RESULTADOS}

\section{Prácticas Administrativas como criterio de informalidad}

Dentro de la literatura revisada no se han encontrado referencias de informalidad empresarial desde un enfoque administrativo, entendiendo esto como estilos de dirección, herramientas, métodos, metodologías, técnicas o actividades que hayan sido incorporados en la administración de las empresas de manera empírica.

Este puede ser un elemento importante de análisis, dado que un elevado porcentaje de propietarios de empresas informales tienen niveles muy bajos de formación o capacitación, y abordan de manera empírica la gestión de éstas (Cerdán, 2010), lo cual puede dar cabida a varios campos de análisis como el efecto de la formación profesional en la supervivencia de empresas, las prácticas administrativas empíricas como elemento innovador en la gestión organizacional y la definición cualitativa y cuantitativa de las buenas y malas prácticas en empresas informales, entre otras.

Bloom, Sadun y Van Reen (2012) hicieron un estudio organizacional, en el que categorizaron las diferentes prácticas de gestión administrativa encontradas en varios países en diferentes continentes. La metodología propuesta por estos autores ofrece la posibilidad de adelantar un estudio similar en Colombia, enfocado en pymes constructoras, para determinar qué prácticas teóricas o empíricas de tipo administrativo o de gestión empresarial se están utilizando en empresas informales en general, y en las del sector de la construcción en particular.

La relevancia que tiene este tipos de estudios van, por un lado, encaminados al enriquecimiento de la teoría administrativa y de gestión con una aproximación fundamentada empíricamente, y por otro, a la medición de la participación real de este tipo de empresas en la empleabilidad, la productividad, y la competitividad nacional.

En cuanto a la informalidad empresarial en Colombia, se identificaron estudios orientados principalmente a la informalidad relacionada con el criterio 3 propuesto por Hamann y Mejía, que hace referencia al no pago de las prestaciones sociales y contribuciones a la nómina. Tal es el caso del estudio hecho por el Consejo Privado de Competitividad, que estableció que la tasa de informalidad laboral en Colombia ascendía al 56,8\% para el año 2012 (CPC, 2014, p. 74), y que la tasa de informalidad laboral específica del sector de la construcción, se encontraba en el $58 \%$, con una productividad relativa del $11 \%$ para esa fecha. (CPC, 2014, p. 75)

En un estudio previo, (Céspedes Rangel, Quintero, Díaz y Galeano, 2011, p. 20), se aproximaron al criterio 2 definido por Hamann y Mejía, entendido como la ausencia del registro mercantil vigente, encontrando que la tasa de informalidad empresarial en el sector construcción para el año 2011 era del $26.5 \%$

Dentro de la literatura revisada, no se encontraron evidencias de estudios relacionados con otros criterios de informalidad empresarial, lo cual brinda una oportunidad interesante para el desarrollo de nuevas investigaciones en este campo, que permitan medir a largo plazo la productividad real no solo del sector de la construcción, sino de otros sectores de reconocida dinámica socioeconómica a nivel nacional. 
LÍNEA DE INVESTIGACIÓN: COMPETITIVIDAD DE EMPRESAS CONSTRUCTORAS

\section{COMENTARIOS}

La informalidad empresarial es un fenómeno que abarca múltiples dimensiones, y se ve clásicamente abordado en la literatura más por el efecto que genera en la evasión de compromisos legales y fiscales, el impacto sobre los índices de empleabilidad en el sector de estudio, o el perfil del gerente o propietario que dirige una empresa informal, que por la necesidad implícita de conocer sus partes componentes, y las características que lo definen desde la óptica administrativa.

Ello hace que sea realmente escasa la literatura que se centra en el análisis de la relación entre informalidad empresarial y prácticas administrativas empíricas, abriendo una ventana de oportunidad para el análisis de la dinámica de la empresa pyme colombiana desde el enfoque administrativo.

Con la literatura revisada, se pudo visualizar que la informalidad empresarial es un fenómeno trasversal que afecta indistintamente sectores, regiones o países, y que por sus características clandestinas, hace difícil la accesibilidad a la participación de ésta en el contexto económico que la rodea. Estudios posteriores se sugieren en este sentido para indagar acerca de dicho fenómeno y sus particularidades en cada sector de la economía Nacional, dada el efecto que esta tiene en dimensiones social política y económica del País.

El fenómeno de informalidad, clásicamente, se ha trabajado a nivel económico como una problemática dicotómica, pero con la revisión académica realizada, se pudo identificar que es un fenómeno con varias categorías, lo cual permitirá en investigaciones posteriores una mayor caracterización de sus partes componentes y sus interacciones, y efectos.

Estudios revisados indican que la informalidad empresarial en Colombia supera el $85 \%$, lo cual tiene significativos efectos sobre factores tales como la tributación en Colombia, y las condiciones laborales de los involucrados. Ello podría indicar que hay elevada tasa de evasión fiscal y una pauperización de las condiciones laborales en los diferentes sectores económicos del país. Se sugieren estudios posteriores a este respecto.

Dentro de la literatura revisada no se encuentran estudios recientes relacionados con algunos tipos de informalidad, y en el caso particular del sector de la construcción, cobra mayor relevancia dado que es un sector que tiene una elevada participación porcentual en el producto interno bruto, y que es un proveedor natural del estado con infraestructura y obras civiles de cobertura regional.

Las prácticas administrativas, definen de manera determinante la orientación estratégica, la toma de decisiones, el uso y asignación de recursos, las formas y tecnologías de producción, y el camino del crecimiento y la permanencia. Es por ello que se propone como nuevo criterio de análisis de informalidad para estudios posteriores en los diferentes sectores.

La gran pregunta, que rige esta investigación y que es frecuentemente discutida en espacios académicos, es ¿por qué empresas con bajos niveles de formación y de apropiación de conocimiento, logran su permanencia y crecimiento en entornos altamente competitivos, y empresas con alto nivel de preparación, presentan aparentemente tan bajos niveles de permanencia en el mercado?

Es por lo anteriormente expuesto, que se considera como una primera hipótesis que las prácticas administrativas, juegan un papel determinante en la permanencia y crecimiento empresarial, independientemente de la condición de formalidad o informalidad que estas presenten. 


\section{REFERENCIAS}

Bloom, N., Genakos, C., Sadun, R. y Van Reenen, J. (2012). Management practices across firms and countries. The Academy of Management Perspectives, 26(1), 12-33.

Cerdán, Á. (2010). Análisis del nivel de profesionalización en la empresa familiar. Revista De Estudios Empresariales. Segunda Época, (2).

Cespedes, E., Quintero, N., Diaz, G. y Galeano, G. (2011). Una mirada a la economía informal.

Chung, K. y Megginson, L. (1981). Organizational behavior: Developing managerial skills New York: Harper \& Row.

Corominas, J. y Pascual, J. A. (1984). 1987. Diccionario Critico Etimologico Castellano E Hispanico. Gredos, Madrid.

Crosby, P. (2011). Dinámica gerencial: El arte de motivar a los demás McGraw-Hill Interamericana.

De Competitividad, C. N. (2014). Informe nacional de competitividad 2013-2014. Bogotá.

Development. Statistics Directorate, International Monetary Fund. Bureau of Statistics, International Labour Organisation. Bureau of Statistics y Commonwealth of Independent States Statistical Committee. (2002). Measuring the non-observed economy: A handbook Lippincott Williams \& Wilkins.

Drucker, P. F. (2008). Management rev ed Zondervan.

Fayol, H. (1947). Administration industrielle et générale: Prévoyance, organisation, commandement, coordination, contrôle Dunod.

Hamann, F., \& Mejía, L. (2011). Formalizando la informalidad empresarial en colombia. Borradores De Economia, (676)

Krygier, A. (1992). La gerencia del futuro. El Universal.

Motta, P. y de Orticochea, L. (1993). La ciencia y el arte de ser dirigente Tercer Mundo Editores, Uniandes.

Pedersen, S. (2003). The shadow economy in germany, great britain and scandinavia: A measurement based on questionnaire surveys Rockwool Foundation Research Unit.

Santa María, S., \& Rozo, V. (2009). Análisis cualitativo y cuantitativo de la informalidad empresarial en colombia. Desarrollo Y Sociedad, (63), 269-296.

Stoner, J., Freeman, R., Sacristan, P. y Gilbert, D. (1996). Administración Prentice Hall.

Taylor, F. y Fayol, H. (1977). Principios de la administración científica. 